\title{
EMPREGO DO ALGORITMO BUSCA TABU NA DETERMINAÇÃO DE PRINCIPIOS ATIVOS EM FÁRMACOS
}

\author{
Pedro Henrique de Almeida Konzen \\ Curso de Matemática Aplicada e Computacional - UNISC - \\ Av. Independência 2293, Santa Cruz do Sul, RS, CEP 96815-900 \\ João Carlos Furtado \\ Departamento de Informática - UNISC - \\ Av. Independência 2293, Santa Cruz do Sul, RS, CEP 96815-900 \\ Claudia Wollmann Carvalho \\ Coordenação dos Laboratórios de Química - UNISC - \\ Av. Independência 2293, Santa Cruz do Sul, RS, CEP 96815-900

\section{Rolf Fredi Molz} \\ Departamento de Informática - UNISC - \\ Av. Independência 2293, Santa Cruz do Sul, RS, CEP 96815-900

\section{e Marco Flores Ferrão} \\ Departamento de Química e Física - UNISC - \\ Av. Independência 2293, Santa Cruz do Sul, RS, CEP 96815-900
}

A new methodology of analysis for the quantification of antihipertensive drugs is provided in this work, using tabu search optimization with infrared spectroscopy by diffuse reflectance Fourier transform (DRIFTS) and partial-least squares regression method $(P L S)$. Firstly, through an experimental planning, samples with the active principle hidrochlorotiazide in starch were prepared, and the standard set of the spectra as well as of the validation samples were gotten in this process. A diffuse reflectance joined to a Nicolet Magna 550 spectrophotometry in the mid-infrared region was used. The examples were developed in a Matlab, spending the real data in shape of Log $(1 / R)$. The calibration was through PLS in order to use it as a pre-processing. The data were used in multiplicative signal correction (MSC). After the best wavelength selection, which results in models with better coefficient regression $\left(R^{2}\right)$, these were improved using routines based on tabu search heuristic method. For the evaluation of the optimization a standard error of validation (SEV) was used. The new technique using DRIFTS/PLS/TS shows an excellent choice for the quality control of the productive process of factories and drugstores that produce or handle these materials in large scale, presenting low time of analysis, no sample destruction and no wastes production.

Keywords: Tabu Search, Pharmacological Formulations, multivariate regression. 


\section{Introdução}

Até recentemente, a espectroscopia na região do infravermelho era pouco usada em análises quantitativas devido a algumas limitações inerentes. Porém, com a utilização da transformada de Fourier na espectroscopia por infravermelho (FT-IR) aliada ao desenvolvimento de técnicas quimiométricas, ao advento dos microprocessadores e de técnicas computacionais avançadas, permitiram a análise de misturas complexas, como fármacos, sem a necessidade de qualquer separação prévia de seus componentes.

Este avanço da espectroscopia no infravermelho médio, como técnica para análise quantitativa, deve-se à combinação da transformada de Fourier e da nova geometria dos espectrofotômetros com a utilização do interferômetro de Michelson [1-4], tornando os espectrofotômetros mais rápidos e robustos.

Por outro lado, técnicas computacionais heurísticas, como os genetic algorithms (GA) o simulated annealing (SA) e a tabu serach (TS), vem sendo cada vez mais empregadas na seleção de variáveis (comprimentos de onda), buscando otimizar os modelos de regressão, tornando-os mais robustos.[5]

Neste trabalho, a meta-heurística denominada busca tabu (tabu search) é empregada para otimizar modelos de regressão multivariados via dados de espectroscopia por reflexão difusa no infravermelho com transformada de Fourier (DRIFTS), aplicando métodos estatísticos de calibração por mínimos quadrados parciais (PLS), na determinação de princípios ativos de ação anti-hipertensiva presentes em fármacos; visando a análise quantitativa da hidroclorotiazida, pertencente à classe diuréticos tiazídicos.

\section{Fármacos Anti-hipertensivos}

Alguns fármacos têm a propriedade de reduzir a pressão atuando na resistência periférica e/ou no débito cardíaco, conhecido como anti-hipertensivos. Entre os antihipertensivos mais empregados encontramos os da classe das Tiazidas, também chamadas derivados da benzotiazina ou benzotiazidas, que são quimicamentes dióxidos de benzotiadiazina-7-sulfonamida[6].

O princípio ativo 6-cloro-3,4-diidro-2H-1,2,4-benzotiodiazina-7-sulfonamida-1,1dióxido, conhecido como hidroclorotiazida, apresenta fórmula estrutural conforme figura 1.

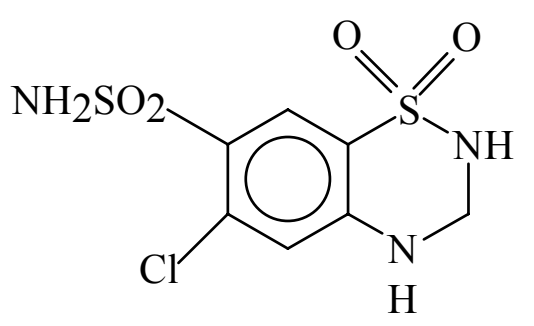

Figura 1: Estrutura da hidroclorotiazida

A hidroclorotiazida apresenta-se como um pó cristalino branco ou quase branco, inodoro e com peso molecular de $297,7 \mathrm{~g} / \mathrm{mol}$. É muito pouco solúvel em água, pouco solúvel em álcool, solúvel em acetona e em soluções diluídas de hidróxidos alcalinos, apresentando faixa de fusão de $266-270^{\circ} \mathrm{C}$, com decomposição.

A determinação de absortividade molar; a obtenção do espectro de absorção no infravermelho; e a técnica de cromatografia planar são ensaios realizados para a identificação da hidroclorotiazida. Já os índices de acidez e/ou alcalinidade são usados na 
determinação da pureza. Já na determinação das impurezas são realizados testes para substâncias relacionadas que são feitos através da técnica de cromatografia em camada delgada.

Os comprimidos de hidroclorotiazida devem conter, no mínimo, 93\% e, no máximo, $107 \%$ da quantidade de hidroclorotiazida declarada. São realizados testes de desintegração, dureza, friabilidade e uniformidade de conteúdo para a caracterização dos mesmos. $\mathrm{O}$ doseamento é realizado através da técnica de espectrofotometria no ultravioleta e por cromatografia líquida de alta eficiência [7].

\section{Reflexão Difusa}

A técnica de reflexão difusa está bem fundamentada na literatura [8-16], sendo amplamente aplicada associada aos equipamentos que operam no infravermelho próximo, com os quais geralmente é designada de espectroscopia de reflexão no infravermelho próximo (NIRS ou NIRR); ou associada àqueles que operam na região do infravermelho médio, sendo conhecida por espectroscopia por reflexão difusa no infravermelho médio com transformada de Fourier (DRIFTS).

A reflexão difusa ocorre em superfícies não totalmente planas, podendo o substrato ser contínuo ou fragmentado (na forma de pó). Neste processo de reflexão o feixe incidente penetra a superfície da amostra interagindo com a matriz, retornando à superfície da mesma, após absorção parcial e múltiplos espalhamentos, conforme ilustrado pela figura 2.

$\mathrm{Na}$ reflexão difusa, a radiação incidente entra em contato diversas vezes com as partículas da amostra sendo conseqüentemente atenuada. Com isto a radiação que sofre a reflexão difusa fornece informações qualitativas e quantitativas, sobre a natureza química da amostra.

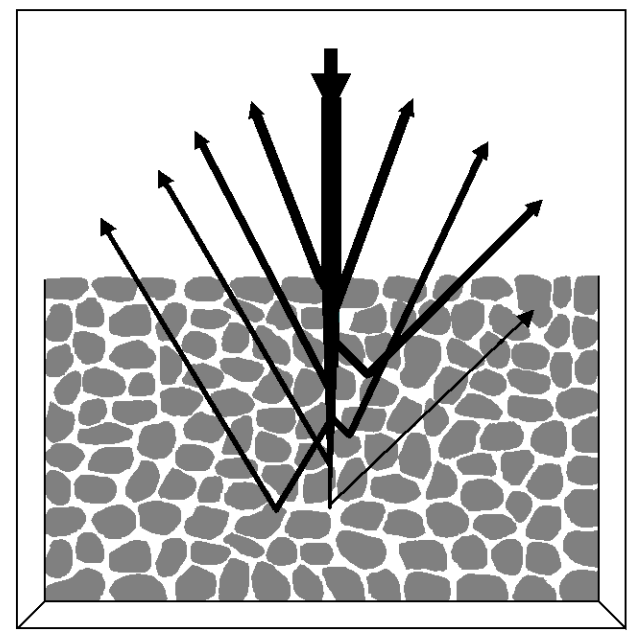

Figura 2: Representação da reflexão especular e difusa de uma onda eletromagnética em

\section{Técnicas Heurísticas de Otimização} uma amostra particulada [15].

Enquanto os problemas lineares contínuos possuem no simplex um algoritmo muito eficiente para a solução exata, os problemas lineares discretos, salvo alguns casos particulares, normalmente não são apropriados para este algoritmo. Desta forma, freqüentemente são propostos algoritmos heurísticos para obter soluções de qualidade. 
Heurística é qualquer método ou técnica criada, ou desenvolvida, para resolver um determinado tipo de problema. As meta-heurísticas são consideradas de uso geral ou uma heurística das heurísticas. Neste trabalho usa-se a heurística denominada Busca Tabu.

\subsection{Busca Tabu}

A Busca Tabu (BT) é um método heurístico, aplicado com grande êxito a um grande número de problemas combinatoriais. A BT foi proposta por Glover [17-18]. A idéia básica consiste em evitar mínimos locais na busca, usando algumas estruturas para soluções (ou movimentos ) proibidas (tabu).

Seja $X$ o conjunto de possíveis soluções e $S(x)$ o conjunto de movimentos que levam de uma solução $x$ para uma outra solução $x$ '. É criado um subconjunto $T$ de $S$, cujos elementos são chamados movimentos tabu. Os elementos de $T$ são determinados por meio de uma função que utiliza informações históricas do processo de busca. Um elemento importante na busca tabu é a incorporação da aspiração $A(f(x))$, critério que permite que um movimento seja realizado mesmo sendo tabu. Uma das características da busca tabu é apresentar-se como uma forma de busca em vizinhança. Desta forma, cada solução $x \in X$, tem um conjunto de vizinhos, $N(x) \subset X$, chamado de vizinhança de $x$. Cada elemento $x$ ' $\epsilon$ $N(x)$ pode ser alcançado diretamente a partir de $x$, mediante um movimento. A qualidade de cada solução $x$ gerada é avaliada mediante o cálculo da função objetivo $f(x)$.

\section{Modelagem Quimiométrica}

Foram definidas através de um planejamento experimental 22 amostras contendo o princípio ativo hidroclorotiazida em amido, das quais, 17 foram usadas para calibração (34 espectros) e 5 para validação (10 espectros). O conjunto de espectros dos padrões e das amostras de validação (figura 3) foram obtidos utilizando-se um acessório de reflectância difusa acoplado a um espectrômetro Nicolet Magna 550 na região do infravermelho médio.

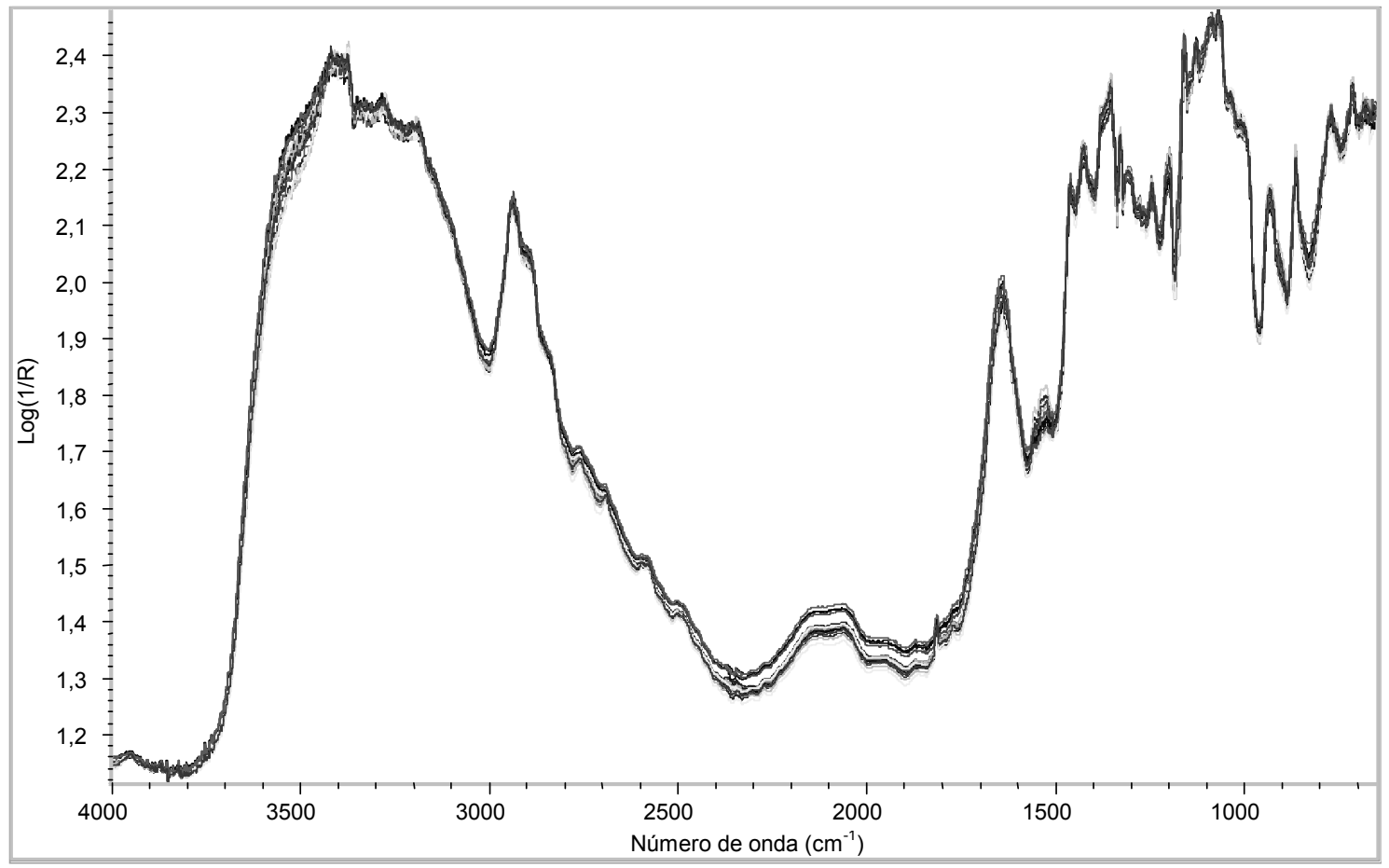

Figura 3: Espetros das amostras de hidroclorotiazida em diferentes concentrações. 
Os modelos foram desenvolvidos em ambiente MATLAB, empregando os dados brutos em forma de $\log (1 / \mathrm{R})$. A calibração foi realizada com o método de regressão por mínimos quadrados parciais (PLS) utilizando a correção do sinal multiplicativo (MSC) ao conjunto de espectros.

As faixas de comprimento de onda compreendendo 959-1799 e 2800-3849 $\mathrm{cm}^{-1}$ foram utilizadas no processo de otimização empregando-se rotina baseada em busca tabu com base na função objetiva $((\mathrm{SEV}+\mathrm{SEC})+|(\mathrm{SEV}-\mathrm{SEC})|)$, onde SEV representa o erro padrão das amostras de validação e SEC o erro padrão das amostras de calibração.

Como parâmetros de entrada da rotina busca tabu foram empregados:

a. tamanho da lista tabu $=20$;

b. percentagem de números de onda selecionados $=5 \%$;

c. percentagem de perturbação dos bits para geração de soluções vizinhas $=20 \%$;

d. número máximo de variáveis latentes $=10$;

e. número de iterações intermediárias $(\mathrm{qmax})=50$;

f. número total de iterações $(\operatorname{pmax})=250$;

g. número de vizinhos $=50$.

\section{Resultados}

$\mathrm{Na}$ tabela 1 são apresentados alguns resultados da otimização empregando a algoritmo de busca tabu, fazendo-se variar o número de variáveis latentes empregadas no modelo de calibração (PLS). Pode-se observar que a partir de 5 variáveis latentes o modelo torna-se estável em relação ao coeficiente de regressão $\left(\mathrm{R}^{2}\right)$, indicando ser este um número suficiente para gerar modelos representativos para a quantificação do fármaco em questão.

\begin{tabular}{cccccc}
\hline $\mathbf{N}^{\mathbf{0}}$ de Variáveis Latentes & SEV $(\%)$ & SEC $(\%)$ & $\mathbf{R}^{2}$ & Variáveis & Tempo(min) \\
\hline 1 & 3,0177 & 4,1387 & 0,5767 & 3 & 3 \\
2 & 1,3835 & 1,3839 & 0,9211 & 22 & 3 \\
3 & 1,1673 & 1,2023 & 0,9405 & 42 & 3 \\
4 & 1,0462 & 1,0426 & 0,9552 & 75 & 7 \\
5 & 0,2880 & 0,2895 & 0,9965 & 112 & 9 \\
6 & 0,1831 & 0,1796 & 0,9987 & 70 & 9 \\
7 & 0,1767 & 0,1872 & 0,9986 & 65 & 8 \\
8 & 0,2438 & 0,2530 & 0,9974 & 73 & 6 \\
9 & 0,2173 & 0,2319 & 0,9978 & 50 & 5 \\
10 & 0,1143 & 0,1489 & 0,9991 & 56 & 6 \\
\hline
\end{tabular}

Tabela 1 - Valores dos parâmetros de acompanhamento da otimização em função do número de variáveis latentes empregados na modelagem PLS.

Para verificar esta tendência foram repetidas mais 4 vezes o conjunto de 250 iterações para os casos onde foram empregadas 4,5 e 6 componentes principais sendo os resultados da otimização da função objetiva semelhantes ao exemplo apresentado na Figura 4. 
Nas figuras 5 e 6 são apresentadas as curvas de calibração para a modelagem PLS empregando 5 variáveis latentes e, respectivamente, todas os números de onda da região espectral estudada e apenas os 112 números de onda selecionados (variáveis) pela busca tabu.

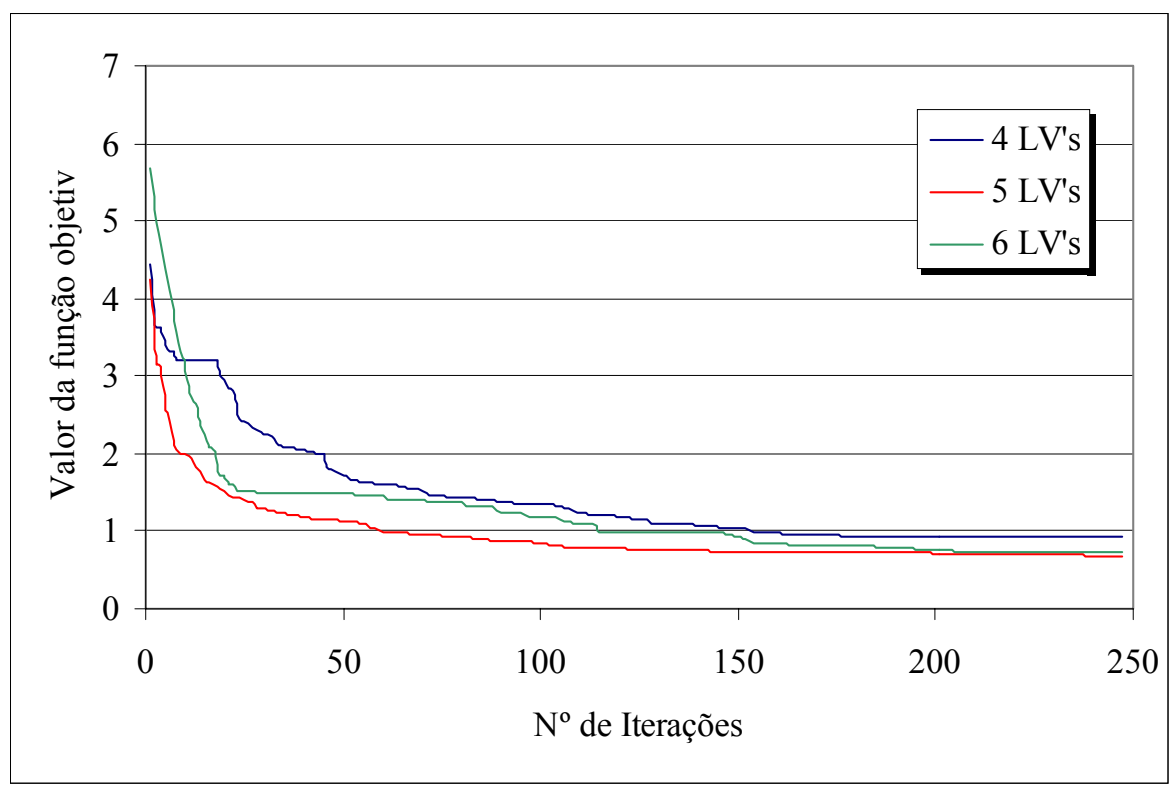

Figura 4 : Representação gráfica dos valores da função objetiva em função do número de iterações empregando a rotina de otimização busca tabu..

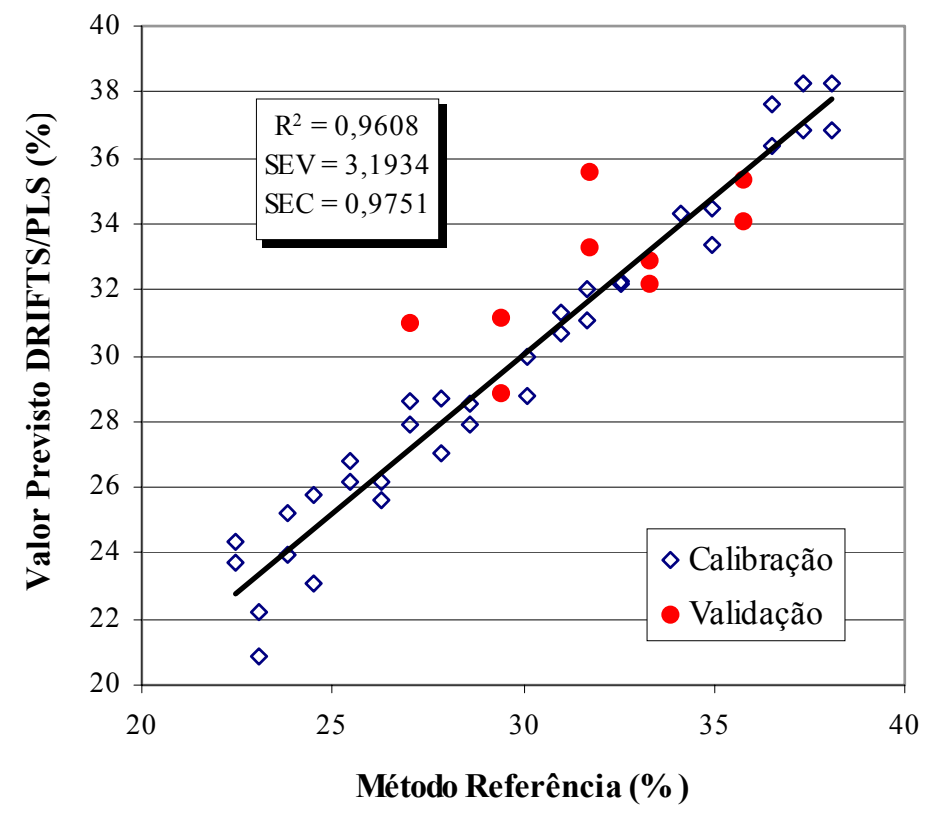

Figura 5: Curva de calibração para o modelo que emprega todos os comprimentos de onda e 5 variáveis latentes. 


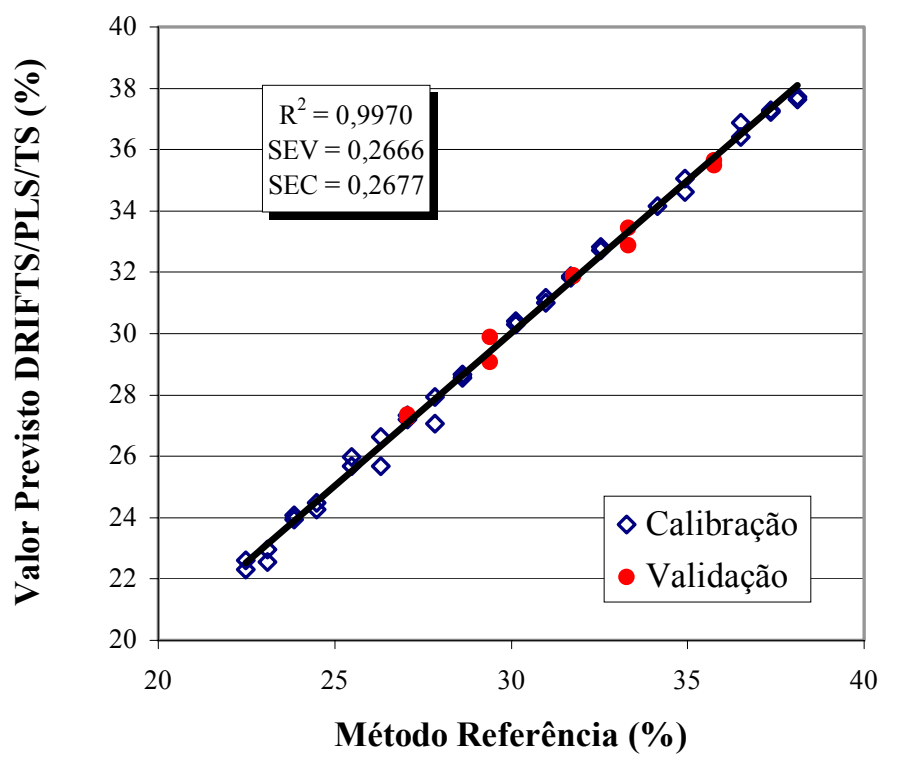

Figura 6: Curva de calibração para o modelo que emprega somente 112 comprimentos de onda selecionados pela busca tabu e 5 variáveis latentes.

\section{Conclusões}

Para todos os modelos otimizados empregando 4, 5 ou 6 variáveis latentes foi observada uma significativa melhora na habilidade de previsão destes, quando são selecionadas os comprimentos de onda dos espectros através da rotina de busca tabu. Este comportamento pode ser explicado, uma vez que esta redução de variáveis (seleção) deve estar sendo acompanhada pela redução do antagonismo entre as diferentes freqüências dos espectros modelados.

Também é observada que a melhoria da capacidade de previsão dos modelos é acompanhada por um acréscimo no coeficiente de correlação das amostras de calibração $\left(\mathrm{R}^{2}\right)$, o que indica uma maior robustez nos modelos onde a busca tabu é empregada.

Um atrativo ao emprego da otimização empregando técnicas meta-heurísticas reside no fato destas serem facilmente implementadas e apresentarem baixo tempo computacional, quando empregas em problemas cuja melhor solução global é inviável de ser alcançada em função do elevado número de combinações possíveis.

Estes resultados demonstram as potencialidades da pesquisa operacional através da técnica DRIFTS/PLS/TS aqui proposta, como uma excelente alternativa para o monitoramento do processo produtivo de empresas e farmácias que produzam ou manipulem estes princípios ativos em larga escala, apresentando baixo tempo de análise, não destruição da amostra e a não geração de resíduos.

\section{Referências Bibliográficas:}

1. KALASINSKY,K.S. - Trend. Anal. Chem., 9, 83-89, 1990.

2. DURIG,J.R. \& SULLIVAN,J.F. - Trend. Anal. Chem., 9, 104-106, 1990.

3. EIKREM,L.O. - Trend. Anal. Chem., 9, 107-109, 1990.

4. COATES,J. - Appl. Spectrosc. Rev., 33, 267-425, 1998.

5. LEARDI, R. - Journal of Chemometrics, 15, 559-569, 2001.

6. HARVEY, R. A.; CHAMPE, P. C. Farmacologia Ilustrada. 2.ed. Porto Alegre: Artmed, 1998. 478p. 
7. FARMACOPÉIA Brasileira. 4.ed. São Paulo: Atheneu, 1988. p. 33,47.

8. WILSON,R.H. - Trend. Anal. Chem., 9, 127-131, 1990.

9. VAN DE VOORT,F.R. - Food Res. Int., 25, 397-403, 1992.

10. HART,J.R.; NORRIS,K.H. \& GOLUMBIC,C. - Cereal Chem., 39, 94-99, 1962.

11. ABDULLAH,A.H. \& SHERMAN,W.F. - Vib. Spec., 13, 133-142, 1997.

12. BIRTH,G.S. \& HECHT,H.G. - The physics of near-infrared reflectance - in Nearinfrared technology in the agricultural and food industries - by Phil Wiliams \& Karl Norris, St. Paul: American Association of Cereal Chemists, 1987.

13. CULLER,S.R. - Diffuse reflectance infrared spectroscopy: Sampling techniques for qualitative/quantitative analysis of solids - in Practical sampling techniques for infrared analysis - by Patricia B. Coleman, Boca Raton: CRC Press, 1993.

14. OSBORNE,B.G.; FEARN,T. \& HINDLE,P.H. - Practical NIR spectroscopy with applications in food and beverage analysis - Singapure: Longman Scientific \& Technical, 1993.

15. WETZEL,D.L. - Anal. Chem., 55, 1165A-1176A, 1983.

16. FERRÃO, M.F. - Tecno-Lógica, 5, 63-85, 2001.

17. GLOVER, F. Tabu Search-part I. ORSA Journal on Computing, 1, 3, 190-206, Summer 1989a.

18. GLOVER, F. Tabu Search - part II. ORSA Journal on Computing, 2, 4-32, Winter 1989b. 